\title{
Infectious diseases in Singapore and Asia: persistent challenges in a new era
}

\author{
Lawrence Soon-U Lee ${ }^{1,2}, \mathrm{MRCP}, \mathrm{PhD}$
}

I nfectious diseases continue to be a major problem in Singapore and Asia, and developments in medical technology have sometimes, paradoxically, contributed to the issue. Although the spotlight has shifted over time from infectious diseases to the chronic, non-infectious diseases that develop in the course of ageing, infectious diseases are still an important cause of illness and death worldwide. ${ }^{(1)}$ In addition to the predictable burden of endemic diseases such as Candida and dengue, the threat of pandemics is ever-present and global, and Singapore and Asia have not been spared from this onslaught.

In this environment, the Singapore Medical Journal (SM) continues to do its part in showcasing research on infectious diseases in the region. This issue highlights articles on the threat of the Zika virus (ZIKV) in the newborn, neurological sequelae of enteroviruses in children and prognostic factors influencing the mortality risk of candidaemia in intensive care unit patients. They contribute to the body of research as the fight against emerging and re-emerging infectious diseases continues into a new era.

In 2013, Singapore commemorated the tenth anniversary of SARS (severe acute respiratory syndrome). Although the nation state has increased its infectious diseases and pandemic preparedness after that significant event, it still suffers from epidemics, such as the H1N1 influenza pandemic in 2009 and the enterovirus infection that resulted in the outbreak of hand, foot and mouth disease (HFMD) in 2008. ${ }^{(2)}$ The ZIKV infection, which Lin et al discuss in their paper, ${ }^{(3)}$ was another such epidemic. On 27 August 2016, a case of locally acquired ZIKV infection was identified in Singapore. Subsequent investigations revealed many more ZIKV infections, with the earliest onset of illness dating as far back as 31 July 2016. More than 200 cases were detected within a week, including in two pregnant women. This marked the official arrival of ZIKV in Singapore, a revelation that could have resulted from the development of better diagnostic techniques. ${ }^{(4,5)}$

Like SARS, which continues to affect its victims long after their recovery from the acute infection, ${ }^{(6-9)}$ ZIKV infection has long-term sequelae, with the development of congenital Zika syndrome in the newborn being the main source of concern. This led to the World Health Organization's declaration that the ZIKV outbreak in South America, and the associated increase in neurological disorders and neonatal malformations, was a "public health emergency of international concern". In their review, Lin et al considered existing case reports and case series to summarise the clinical features of ZIKV infection. They also reviewed the risk of congenital infections in Singapore and the measures taken to monitor and prevent this devastating sequela. ${ }^{(3)}$

Enterovirus infection is another common, endemic infectious disease in Singapore with occasional large outbreaks. ${ }^{(2)}$ The cause of the well-known HFMD, enterovirus infection may have severe neurological sequelae in children. Taking into account the lack of previous studies on neurological manifestations, Thong et al's article reviewed the clinical virology of enteroviruses in Singaporean children. ${ }^{(10)}$ The case-control study was performed in a single centre and included 48 children with confirmed enterovirus infection. A high percentage $(75 \%)$ of the patients had neurological manifestations, mostly aseptic meningitis. An unfortunate finding was that long-term neurological sequelae were seen in $16.7 \%$ of the patients. This study underscores the need to evaluate for neurological involvement in children with this common infection.

A disease that is common among intensive care unit patients in Asia and the developed world is candidaemia, which carries a poor prognosis. With the development of modern medical technology such as critical care, innovative surgeries and cancer chemotherapy, the number of patients at risk of candidaemia has increased. Kawano et al's article reviewed 25 patients with candidaemia in a tertiary referral centre in Japan; of these, 18 patients died. The researchers identified the Sequential Organ Failure Assessment score and cumulative number of risk factors for invasive candidiasis as variables that predict death due to candidaemia. ${ }^{(11)}$ Risk factors for candidaemia, such as the use of broad-spectrum antimicrobial agents (i.e. carbapenems or tazobactam/piperacillin), have not changed over time. This study highlights the need to monitor and reduce risk factors for candidaemia, and brings up the possible role of early initiation of antifungal therapy to decrease in-hospital mortality.

Infectious diseases remain a major burden for mortality and morbidity in Singapore and the rest of Asia. There is a need to be vigilant for pandemics such as ZIKV and SARS. At the same time, we need to manage ongoing endemic diseases such as enteroviruses and dengue, and control diseases contributed by improving medical technologies, such as candidaemia. More research needs to be done in these areas to prevent these infectious diseases and improve clinical care.

\section{REFERENCES}

1. Dye C. After 2015: infectious diseases in a new era of health and development. Philos Trans R Soc Lond B Biol Sci 2014; 369:20130426.

2. Wu Y, Yeo A, Phoon MC, et al. The largest outbreak of hand; foot and mouth disease in Singapore in 2008: the role of enterovirus 71 and

\footnotetext{
${ }^{1}$ Yong Loo Lin School of Medicine, National University of Singapore, ${ }^{2}$ Department of Medicine, National University Health System, Singapore Correspondence: Dr Lawrence Soon-U Lee, Assistant Professor, Department of Medicine, National University Health System, NUHS Tower Block Level 10, 1E Kent Ridge Road, Singapore 119228. lawrence_lee@nuhs.edu.sg
} 
coxsackievirus A strains. Int J Infect Dis 2010; 14:e1076-81.

3. Lin HZ, Tambyah PA, Yong EL, Biswas A, Chan SY. A review of Zika virus infections in pregnancy and implications for antenatal care in Singapore. Singapore Med J 2016; 58:171-8.

4. Leo YS, Chow A. Zika virus has arrived in Singapore. Lancet Infect Dis 2016; 16:1317-9.

5. Lysaght T, Lee TL, Watson S, et al. Zika in Singapore: insights from One Health and social medicine. Singapore Med J 2016; 57:528-9.

6. Kwek SK, Chew WM, Ong KC, et al. Quality of life and psychological status in survivors of severe acute respiratory syndrome at 3 months postdischarge. J Psychosom Res 2006; 60:513-9.

7. Ong KC, Ng AW, Lee LS, et al. Pulmonary function and exercise capacity in survivors of severe acute respiratory syndrome. Eur Respir J 2004;
24:436-42.

8. Ong KC, Ng AW, Lee LS, et al. 1-year pulmonary function and health status in survivors of severe acute respiratory syndrome. Chest 2005; 128:1393-400.

9. Leow MK, Kwek DS, Ng AW, et al. Hypocortisolism in survivors of severe acute respiratory syndrome (SARS). Clin Endocrinol (Oxf) 2005; 63:197-202.

10. Thong WY, Han A, Wang SJ, et al. Enterovirus infections in Singaporean children: an assessment of neurological manifestations and clinical outcomes. Singapore Med J 2016; 58:189-95.

11. Kawano Y, Togawa A, Nakamura Y, et al. Prognostic factors for candidaemia in intensive care unit patients: a retrospective analysis. Singapore Med J 2016; 58:196-200. 